\title{
ALGEBRAIC CYCLES AND THE LIE ALGEBRA OF MIXED TATE MOTIVES
}

\author{
SPENCER BLOCH
}

The purpose of this note is to suggest a candidate for the category $\mathscr{M} \mathscr{M}=$ $\mathscr{M} \mathscr{M}_{F}$ of mixed Tate motives $(\otimes \mathbb{Q})$ over a field $F$. We will define a graded pro-Lie algebra $\mathscr{L}=\mathscr{L}_{-1} \oplus \mathscr{L}_{-2} \oplus \cdots$ defined over $\mathbb{Q}$ and depending on $F$. $\mathscr{M} \mathscr{M}$ will be the category of f.d. graded $\mathbb{Q}$-representations of $\mathscr{L}$. To avoid having to work with pro-objects, we consider the co-Lie algebra $\mathscr{M}=\mathscr{L}^{v}$ (continuous dual). $\mathscr{M}$ is graded in positive degrees and is generated by suitable algebraic cycles. To link $\mathscr{M}$ to the category of mixed Tate motives, we will verify the following properties:

(i) $\mathscr{M}_{1}=F^{\times} \otimes \mathbb{Q}$, where $F^{\times}$is the multiplicative group of $F$.

(ii) $\mathscr{M} \supset \mathscr{M}_{\text {log }}$, where $\mathscr{M}_{\log }$ is the co-Lie algebra associated with the polylogarithm functions by Deligne [3]. In particular, the polylogarithm motives defined by Beilinson exist in our theory.

(iii) Suppose $F=\mathbb{C}(V)$, the field of functions on a variety $V$. Let $\mathscr{M}_{\text {top }}$ be the complex 1-minimal model [6, 7] for the de Rham complex of $F$. We define a homomorphism of co-Lie algebras

$$
\varphi: \mathscr{M} \rightarrow \mathscr{M}_{\text {top }}
$$

The theory of minimal models yields $\mathscr{M}_{\text {top }}=\bigcup \mathscr{M}_{\text {top }}(i)$, where $\mathscr{M}_{\text {top }}(i)$ is obtained from $\mathscr{M}_{\text {top }}(i-1)$ by a Hirsch extension. We get

$$
\varphi\left(\bigoplus_{j \leq i} \mathscr{M}_{j}\right) \subset \mathscr{M}_{\mathrm{top}}(i) .
$$

The composition

$$
F^{\times} \otimes \mathbb{Q} \cong \mathscr{M}_{1} \hookrightarrow H^{1}(\mathscr{M}) \stackrel{\varphi}{\rightarrow} H^{1}\left(\mathscr{M}_{\text {top }}\right) \cong H^{1}\left(\Omega^{\cdot}\right)
$$

is given by $f \mapsto d f / f$.

Roughly speaking, (i.e., ignoring problems with basepoints and limits, which means that in real life one must deal with open varieties $V-D$ rather than $\operatorname{Spec}(\mathbb{C}(V)))$ one has $\mathscr{M}_{\text {top }}(i)$ dual to the $\mathbb{C}$-Lie algebra of $\pi_{1}(\operatorname{Spec}(\mathbb{C}(V))) / \pi_{1}^{(i+1)}$ with $\pi_{1}^{(i+1)}=(i+1)$ st step in the descending central series, i.e., $\pi_{1}^{(2)}=\left[\pi_{1}, \pi_{1}\right]$

Received by the editors March 12, 1991.

1991 Mathematics Subject Classification. Primary 14C25, 18 F25. 
and $\pi_{1}^{(i+1)}=\left[\pi_{1}^{(i)}, \pi_{1}\right]$. Dualizing, one gets with obvious notation

$$
\varphi^{\vee}: \mathscr{L}_{\text {top }} \rightarrow \mathscr{L} \otimes \mathbb{C}
$$

so a representation of $W$ of $\mathscr{L}$ gives rise to a representation $\varphi^{*}(W)$ of $\mathscr{L}_{\text {top }}$, i.e., an isomorphism class of $\mathbb{C}$-local systems defined on some $V-D$ and having nilpotent monodromy. If our motivic interpretation of $\mathscr{L}$ is correct, such a $W$ is a mixed Tate motive. Its Hodge realization is a nilpotent variation of mixed Tate Hodge structures defined on some open $V-D$, and $\varphi^{*}(W)$ should be the underlying local system of $\mathbb{C}$-vector spaces. One may look instead of the $\mathbb{Q}$-coLie algebra $\mathscr{M}_{\mathrm{HT}}$ corresponding to the Tannakian category of Zariski germs of variations of Tate mixed Hodge structure over $V$. Passing to the underlying $\mathbb{C}$-local system gives a homomorphism $\mathbb{C} \otimes \mathscr{M}_{\mathrm{HT}} \rightarrow \mathscr{M}_{\text {top }}$, and I expect there should be a lifting $\rho: \mathscr{M} \rightarrow \mathscr{M}_{\mathrm{HT}}$ of $\varphi$. Can one give a construction of $\mathscr{M}_{\mathrm{HT}}$ (or $\mathbb{C} \otimes \mathscr{M}_{\mathrm{HT}}$ ) in the spirit of minimal models, starting from the de Rham complex?

(iv) Finally, the construction of $\mathscr{L}$ is closely related to the interpretation of algebraic $K$-theory in terms of algebraic cycles suggested in [8,9]. It would be of considerable interest to understand this relation more precisely.

It should be noted that this work has been greatly influenced by the ideas of Beilinson, Deligne, and Goncharov.

\section{INTRODUCTION}

The notion of motive was first introduced by A. Grothendieck as a sort of universal Tannakian category associated to the category of smooth projective varieties over a field $k$. Cohomology theories like Betti, de Rham, or étale cohomology could be interpreted as fibre functors from the category of motives to vector spaces. Understanding the "Galois group" of this category seems difficult. The expected semisimplicity of these pure motives was reduced by Grothendieck to some "standard conjectures" about algebraic cycles, but these conjectures have proven intractible.

More recently, work on regulator maps in higher $K$-theory has focused interest on mixed Tate motives. Imagine given a field $F$ and a candidate $\mathscr{M} \mathscr{T} \mathscr{M}$ for the category of mixed Tate motives $(\otimes \mathbb{Q})$ over $F$. As a guide, the reader may think of $F$ as the function field of a variety $V$ over $\mathbb{C}$ and $\mathscr{M} \mathscr{M}$ as a sort of refinement of the category $\mathscr{M} \mathscr{H}$ of Zariski germs of variations of mixed Tate $\mathbb{Q}$-Hodge structures. Semisimple objects in $\mathscr{M} \mathscr{M}$ are the $\mathbb{Q}(n), n \in \mathbb{Z}$. Objects $M$ have an increasing weight filtration $W_{r} M$ with $\mathrm{gr}_{-2 n}^{W} M=\bigoplus \mathbb{Q}(n)$ and $\operatorname{gr}_{-2 n+1}^{W} M=(0)$. One has $\operatorname{Hom}(\mathbb{Q}(n), \mathbb{Q}(m))=(0)$ if $m \neq n$ and $\mathbb{Q}$ otherwise, and the functor

$$
\begin{aligned}
\phi=\bigoplus \phi_{n}: \mathscr{M} \mathscr{T} M & \rightarrow\{\text { f.d. graded } \mathbb{Q} \text {-vector spaces }\} \\
\phi_{-n}(M) & =\operatorname{Hom}\left(\mathbb{Q}(n), \operatorname{gr}_{-2 n}^{W} M\right)
\end{aligned}
$$

is a fibre functor [10]. 
Lemma (0.2). Let $l$ be a $\mathbb{Q}$-linear endomorphism of the functor $\phi$. For $M \in$ $\mathrm{ob}(\mathscr{M} \mathscr{M})$, let $l_{M}: \phi(M) \rightarrow \phi(M)$ and write $l_{M}=\sum l_{M, i}$ where $l_{M, i}$ is homogeneous of degree $i$ as a map of the graded vector space $\phi(M)$. Then $l_{M, i}=0$ for $i>0$.

Proof. Fix $i>0$ and $n$. To see $l_{M, i}: \phi_{n} \rightarrow \phi_{n+i}$ is zero, let $\psi: W_{-2 n} M \rightarrow M$ be the natural inclusion. The lemma follows from the commutative square

$$
\begin{array}{cc}
\phi_{n}\left(W_{2 n} M\right) \stackrel{l_{W_{2 n} M, i}}{\longrightarrow} & \phi_{n+i}\left(W_{2 n} M\right)=(0) \\
\downarrow \phi_{n}(\psi)=\mathrm{id} & \downarrow_{\phi_{n+i}}(\psi) \\
\phi_{n}(M) \stackrel{l_{M, i}}{\longrightarrow} & \phi_{n+i}(M) .
\end{array}
$$

For $M \in \mathrm{Ob}(\mathscr{M} \mathscr{M})$, let $\langle M\rangle$ be the full subcategory of $\mathscr{M} \mathscr{M}$ whose objects are subquotients of $\bigoplus_{N} M$ for some $n$. Rather than considering endomorphisms $l$ of $\phi$ directly, it works better (cf. [10]) to think of $l$ as an endomorphism of $\phi \mid\langle M\rangle$ determined by its action on the finite-dimensional vector space $\phi(M)$. Since $\langle M\rangle \subset\left\langle M \oplus M^{\prime}\right\rangle$, one can (and we will) think of $l$ as a pro-endomorphism.

(0.3) Define $\mathscr{L}$ to be the pro-vector space (in the above sense) of $\mathbb{Q}$-linear endomorphisms of the functor $\phi$ satisfying

(i) For $l \in \mathscr{L}$ and $M, N \in \mathrm{Ob}(\mathscr{M} \mathscr{M})$,

$$
l_{M \otimes N}=\mathrm{id}_{M} \otimes l_{N}+l_{M} \otimes \mathrm{id}_{N}: \phi(M \otimes N) \cong \phi(M) \otimes \phi(N) \rightarrow \phi(M \otimes N),
$$

(ii) $\operatorname{gr}(l): \operatorname{gr}(\phi(M)) \rightarrow \operatorname{gr}(\phi(M))$ is the zero map.

Note (ii) makes sense, since by the lemma, $l$ preserves the filtration fil $_{n} \phi=$ $\bigoplus_{m \leq n} \phi_{m}$.

Lemma (0.4). $\mathscr{L}=\mathscr{L}_{-1} \oplus \mathscr{L}_{-2} \oplus \cdots$ is graded in negative degrees and is a pro-Lie algebra under associative bracket $\left[l_{1}, l_{2}\right]=l_{1} l_{2}-l_{2} l_{1}$ of endomorphisms. (We do not introduce signs in the bracket. If one wants to think of $\mathscr{L}$ as a graded Lie algebra in the usual sense, one should double all degrees to make them even.)

Proof. The only thing to be checked is that (i) is stable under associative bracket, which is straightforward. Q.E.D.

From the abstract theory of Tannakian categories, one has that $\phi$ identifies $\mathscr{M} \mathscr{M}$ with the category of finite-dimensional graded representations of the graded pro-Lie algebra

$$
\mathscr{L}=\mathscr{L}_{-1} \oplus \mathscr{L}_{-2} \oplus \cdots .
$$

In fact, $\mathscr{L}$ is the pro-Lie algebra associated with the nilpotent radical of the Galois group (= automorphism group of the fibre functor) of $\mathscr{M} \mathscr{M}$. It will be convenient to work with the linear dual

$$
\mathscr{M} \stackrel{\text { def }}{=} \mathscr{L}^{\vee}=\mathscr{M}_{1} \oplus \mathscr{M}_{2} \oplus \cdots \text {. }
$$


Strictly speaking, $\mathscr{M}_{i}$ is an ind-object, but we will take the direct limit and view the $\mathscr{M}_{i}$ as vector spaces. The dual of the bracket is a map

$$
\partial: \mathscr{M} \rightarrow \bigwedge^{2} \mathscr{M}
$$

which is homogeneous of degree 0 . The map $\partial$ can be extended to a derivation of $\bigwedge^{\circ} \mathscr{M}$, and the Jacobi identity is equivalent to

$$
0=\partial \circ \partial: \mathscr{M} \rightarrow \bigwedge^{3} \mathscr{M}
$$

so $\left(\bigwedge^{\bullet} \mathscr{M}, \partial\right)$ is a graded, differential graded algebra.

\section{Construction of}

Write $X=\mathbb{P}_{F}^{1}-\{1\}, X^{s}=X \times \cdots \times X$ (s times). Faces on $X^{s}$ are obtained by setting various coordinate functions $=0$ or $\infty$. An algebraic cycle $Z$ on $X^{s}$ is said to be admissible if, for every face $X^{t} \subset X^{s}$, the intersection $Z \cdot X^{t}$ is defined, and one has $Z \cdot X^{t}=\sum n_{i} W_{i}$ with $n_{i} \in \mathbb{Z}$ and $W_{i}$ absolutely irreducible as a variety over $F$. For example, a zero cycle $\sum n_{i} Z_{i}$ is admissible if the $Z_{i}$ are closed points defined over $F$, none of which lie on a face. Notice that if $Z_{1} \times Z_{2} \subset X^{s_{1}} \times X^{s_{2}}$ is admissible, then both the $Z_{i}$ are admissible.

The symmetric group $\mathscr{S}_{s}$ acts on $X^{s}$. Let $\mathscr{V}^{r}(s)$ denote the group of admissible codimension $r$ algebraic cycles with $\mathbb{Q}$-coefficients on $X^{s}$ that are alternating with respect to the action of $\mathscr{S}_{s}$. Define

$$
\partial=\sum(-1)^{i-1}\left(\partial_{i}^{0}-\partial_{i}^{\infty}\right)
$$

where $\partial_{i}^{j}$ is the restriction map on cycles obtained by setting the $i$ th coordinate function $=j$. The following lemma shows that $\partial$ preserves alternating representations so we have $\partial: \mathscr{V}^{r}(s) \rightarrow \mathscr{V}^{r}(s-1)$.

Lemma (1.1). Let $\mathscr{Z}^{r}(s)$ be admissible cycles of codimension $r$ on $X^{s}$ with no condition of alternation. Let

$$
\text { alt }=1 / s ! \sum \operatorname{sgn}(\sigma) \sigma: \mathscr{Z}^{r}(s) \rightarrow \mathscr{V}^{r}(s) \subset \mathscr{Z}^{r}(s)
$$

be the projection. Then

$$
\partial \circ \text { alt }=\text { alt } \circ \partial .
$$

Proof. Given $\sigma \in \mathscr{S}_{s}$ and $1 \leq i \leq s$, let $R(\sigma, i) \in \mathscr{S}_{s-1}$ be the permutation $\psi_{\sigma(i)}^{-1} \circ \sigma \circ \psi_{i}$ where $\psi_{i}:\{1, \ldots, s-1\} \rightarrow\{1, \ldots, s\}$ omits $i$ and preserves order. Writing $Z_{i}$ for the basis of $\mathbb{Q}^{s}$, one has

$$
\begin{aligned}
& \operatorname{sgn}(R(\sigma, i))=Z_{\sigma(1)} \wedge \cdots \wedge \hat{Z}_{\sigma(i)} \wedge \cdots \wedge Z_{\sigma(s)} / Z_{1} \wedge \cdots \wedge \hat{Z}_{\sigma(i)} \wedge \cdots \wedge Z_{s} \\
& \quad=Z_{\sigma(i)} \wedge Z_{\sigma(1)} \wedge \cdots \wedge \hat{Z}_{\sigma(i)} \wedge \cdots \wedge Z_{\sigma(s)} / Z_{\sigma(i)} \wedge Z_{1} \wedge \cdots \wedge \hat{Z}_{\sigma(i)} \wedge \cdots \wedge Z_{s} \\
& \quad=(-1)^{i-\sigma(i)} \operatorname{sgn}(\sigma) .
\end{aligned}
$$

Writing $\partial_{i}=\partial_{i}^{0}-\partial_{i}^{\infty}$ and $\sigma^{*}$ for pullback of cycles under $\sigma$, one has

$$
\partial_{i} \sigma^{*}=R(\sigma, i)^{*} \partial_{\sigma(i)} \text {. }
$$


Hence

$$
\begin{aligned}
\partial(\operatorname{alt}(Z)) & =1 / s ! \sum_{i=1}^{s} \sum_{\sigma \in \mathscr{S}_{s}}(-1)^{i-1} \partial_{i} \operatorname{sgn}(\sigma) \sigma^{*} Z \\
& =1 / s ! \sum_{(-1)^{\sigma(i)-1} \operatorname{sgn}(R(\sigma, i)) R(\sigma, i)^{*} \partial_{\sigma(i)} Z} \\
& =1 / s ! \sum_{\sigma, j}(-1)^{j-1} \operatorname{sgn}\left(R\left(\sigma, \sigma^{-1}(j)\right)\right) R\left(\sigma, \sigma^{-1}(j)\right)^{*} \partial_{j} Z .
\end{aligned}
$$

Given $\tau \in \mathscr{S}_{s-1}$ and $j, k$, there is a unique $\sigma \in \mathscr{S}_{s}$ such that $R(\sigma, k)=\tau$ and $j=\sigma(k)$. Hence

$$
\partial(\operatorname{alt} Z)=1 /(s-1) ! \sum_{j, \tau \in \mathscr{S}_{s-1}} \operatorname{sgn}(\tau) \tau^{*} \partial_{j} Z=\operatorname{alt}(\partial Z) .
$$

This proves Lemma (1.1). Q.E.D.

Define a graded group $\mathscr{N}^{i}=\bigoplus_{r} \mathscr{V}^{r}(2 r-i)$ and a complex $\mathscr{N}^{\cdot}$, which is $\mathscr{N}^{i}$ in degree $i$ with boundary $\partial: \mathscr{N}^{i} \rightarrow \mathscr{N}^{i+1}$ induced by $\partial$ on $\mathscr{V}^{r}(s)$. One has a product structure $\mathscr{V}^{r}(s) \times \mathscr{V}^{t}(u) \rightarrow \mathscr{V}^{r+t}(s+u)$ given by obvious (external) product of cycles composed with projection on the alternating representation. This induces a graded product structure $\mathscr{N}^{i} \times \mathscr{N}^{j} \rightarrow \mathscr{N}^{i+j}$.

Lemma (1.2). $\mathscr{N}^{\cdot}$ is a graded commutative differential graded algebra. (Note $\mathscr{N}^{i} \neq(0)$ for $i<0$.)

Proof. The product in $\mathscr{N}^{\cdot}$ is given by $Z, W \mapsto \operatorname{alt}(Z \times W)$, with alt as above. Suppose $Z \in \mathscr{V}^{r}(2 r-i) \subset \mathscr{N}^{i}$ and $W \in \mathscr{V}^{s}(2 s-j) \subset \mathscr{N}^{j}$. Since $\mathscr{N}^{i}=$ $\bigoplus_{r} \mathscr{V}^{r}(2 r-i)$, switching $Z$ and $W$ changes the sign by $(-1)^{(2 r-i)(2 s-j)}=$ $(-1)^{\operatorname{deg}(Z) \operatorname{deg}(W)}$, making $\mathscr{N}^{\cdot}$ graded commutative. For associativity we need $\operatorname{alt}(T \times \operatorname{alt}(Z \times W))=\operatorname{alt}(\operatorname{alt}(T \times Z) \times W)$ where $T, Z$, and $W$ are alternating cycles. For cycles $A$ and $B$ (not necessarily alternating) one has $\operatorname{alt}(A \times B)=$ alt (alt $A \times$ alt $B)$, so it suffices to note that $T \times(Z \times W)=(T \times Z) \times W$, which is clear. This proves Lemma (1.2). Q.E.D.

Define $\mathscr{N}^{0+}=\operatorname{Ker}\left(\mathscr{N}^{0} \rightarrow \mathscr{V}^{0}(0)\right)$. Let $J=\left[\bigoplus_{i<-1} \mathscr{N}^{i} \oplus \mathscr{N}^{0+}\right] \cdot \mathscr{N}^{\bullet} . J$ is a graded ideal that is not stable under $\partial$. Let $\mathcal{J}$ be the differential graded ideal generated by $J \cdot \mathscr{J}$ is generated as an ideal by $\bigoplus_{i \leq-1} \mathscr{N}^{i} \oplus \mathscr{N}^{0+} \oplus \partial \mathscr{N}^{0+}$ because $\mathscr{V}^{0}(p)=(0)$ for $p \neq 0$ so $\partial \mathscr{N}^{-1} \subset \mathscr{N}^{0+}$. In particular, the ideal $\mathscr{J} / J \subset \mathscr{N} / J$ is generated by elements of degree 1 .

Lemma (1.3). Let $\mathscr{A}=\mathscr{A}^{1} \oplus \mathscr{A}^{2} \oplus \cdots$ be a graded $k$-algebra (associative and graded commutative without unit) where $k$ is a field. Let $\mathscr{B}=\mathscr{A} / \mathscr{I}$ be a graded quotient algebra, and suppose $\mathscr{B}$ is given the structure of a differential graded algebra; i.e., we are given $\partial: \mathscr{B}^{i} \rightarrow \mathscr{B}^{i+1}$ satisfying the usual identities. Assume

(a) the ideal $\mathscr{I}$ is generated by $\mathscr{I}^{1}$, and

(b) $\bigwedge^{2} \mathscr{A}^{1} \subset \mathscr{A}^{2}$. 
Then

$$
\bigwedge^{2} \mathscr{B}^{1} \subset \mathscr{B}^{2}
$$

Define $\mathscr{M}=\left\{r \in \mathscr{B}^{1} \mid \partial r \in \bigwedge^{2} \mathscr{B}^{1} \subset \mathscr{B}^{2}\right\}$. Assume further that

(c) the map

$$
\left(\mathscr{A}^{2} / \bigwedge^{2} \mathscr{A}^{1}\right) \otimes \mathscr{A}^{1} \rightarrow \mathscr{A}^{3} / \bigwedge^{3} \mathscr{A}^{1}
$$

induced by multiplication is injective. Then

$$
\partial(\mathscr{M}) \subset \bigwedge^{2} \mathscr{M} \subset \bigwedge^{2} \mathscr{B}^{1} \subset \mathscr{B}^{2} .
$$

Proof. (a) implies that $\operatorname{Ker}\left(\bigwedge^{2} \mathscr{A}^{1} \rightarrow \bigwedge^{2} \mathscr{B}^{1}\right)$ surjects onto $\operatorname{Ker}\left(\mathscr{A}^{2} \rightarrow \mathscr{B}^{2}\right)$, so (1.3.1) follows from (b). Similarly, (a) implies that

$$
\begin{aligned}
\operatorname{Ker}\left[\left(\mathscr{A}^{2} / \bigwedge^{2} \mathscr{A}^{1}\right) \otimes \mathscr{A}^{1}\right. & \left.\rightarrow\left(\mathscr{B}^{2} / \bigwedge^{2} \mathscr{B}^{1}\right) \otimes \mathscr{B}^{1}\right] \\
& \rightarrow \operatorname{Ker}\left[\mathscr{A}^{3} / \bigwedge^{3} \mathscr{A}^{1} \rightarrow \mathscr{B}^{3} / \bigwedge^{3} \mathscr{B}^{1}\right]
\end{aligned}
$$

so (c) implies

$$
\left(\mathscr{B}^{2} / \bigwedge^{2} \mathscr{B}^{1}\right) \otimes \mathscr{B}^{1} \hookrightarrow \mathscr{B}^{3} / \bigwedge^{3} \mathscr{B}^{1} .
$$

We are thus reduced to verifying (1.3.2) for a DGA $\mathscr{B}$ satisfying (1.3.1) and (1.3.3). Choose a complement $\mathscr{P}$ so $\mathscr{B}^{1} \cong \mathscr{M} \oplus \mathscr{P}$. We have

$$
\bigwedge^{2} \mathscr{B}^{1} \cong \bigwedge^{2} \mathscr{M} \oplus(\mathscr{P} \otimes \mathscr{M}) \oplus \bigwedge^{2} \mathscr{P}
$$

One has (induced by $\partial$ )

$$
\bar{\partial}: \mathscr{P} \hookrightarrow \mathscr{B}^{2} / \bigwedge^{2} \mathscr{B}^{1}
$$

Choose a splitting

$$
\rho: \mathscr{B}^{2} / \bigwedge^{2} \mathscr{B}^{1} \rightarrow \mathscr{P},
$$

so $\rho \circ \partial=\mathrm{id}_{\mathscr{P}}$. Consider the maps

$$
\begin{gathered}
\bar{\partial} \otimes 1: \mathscr{P} \otimes \mathscr{M} \rightarrow\left[\mathscr{B}^{2} / \bigwedge^{2} \mathscr{B}^{1}\right] \otimes \mathscr{M}, \\
\hat{\partial}: \bigwedge^{2} \mathscr{P} \rightarrow\left[\mathscr{B}^{2} / \bigwedge^{2} \mathscr{B}^{1}\right] \otimes \mathscr{P}, \quad \hat{\partial}\left(p \wedge p^{\prime}\right)=\bar{\partial}(p) \otimes p^{\prime}-\bar{\partial}\left(p^{\prime}\right) \otimes p .
\end{gathered}
$$

Clearly $\bar{\partial} \otimes 1$ is injective. Also $\rho \otimes 1 \circ \hat{\partial}: \bigwedge^{2} \mathscr{P} \rightarrow \mathscr{P} \otimes \mathscr{P}$ is the injective map $p \wedge p^{\prime} \mapsto p \otimes p^{\prime}-p^{\prime} \otimes p$, so $\hat{\partial}$ is injective also. Let $r \in \mathscr{M}$, and write $\partial r=a+b+c$ according to the decomposition (1.3.4) above. Let $\alpha$ denote the $\operatorname{map}(1.3 .3)$. One has $0=\partial \partial r \equiv \alpha[(\bar{\partial} \otimes 1)(b) \oplus \hat{\partial}(c)]\left(\bmod \wedge^{3} \mathscr{B}^{1}\right)$. From the injectivity of $\bar{\partial} \otimes 1, \hat{\partial}$, and $\alpha$ one concludes $b=c=0$ so $\partial \mathscr{M} \subset \wedge^{2} \mathscr{M}$ as claimed. Q.E.D.

We want to apply the lemma with $\mathscr{A}^{i}=(\mathscr{N} / J)^{i}$ and $\mathscr{I}=\mathscr{J} / J$, and it will suffice to verify conditions (b) and (c) above. 
Lemma (1.4). Let $X$ and $S$ be sets, with $S$ finite. Let $Y \subset X^{S}$ be a subset. Let $S=S_{1} \amalg \cdots \amalg S_{p}$ and $S=S_{1}^{\prime} \amalg \cdots \amalg S_{q}^{\prime}$ be partitions of $S$, and let $S_{i j}=S_{i} \cap S_{j}^{\prime}$. Consider the diagram of projections

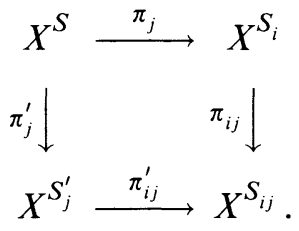

Suppose one has $Y_{i} \subset X^{S_{i}}$ such that $Y=\prod \pi_{i}^{-1}\left(Y_{i}\right)$ and $Y_{j}^{\prime} \subset X^{S_{j}^{\prime}}$ such that $Y=\prod \pi_{j}^{\prime-1}\left(Y_{j}^{\prime}\right)$. Then there are $Y_{i j} \subset X^{S_{i j}}$ such that $Y_{i}=\prod_{j} \pi_{i j}^{-1}\left(Y_{i j}\right)$ and $Y_{j}^{\prime}=\prod_{i} \pi_{i j}^{\prime-1}\left(Y_{i j}\right)$.

Proof. Set theory.

For $z$ irreducible with $\operatorname{alt}(z) \in \mathscr{N}^{0+} \oplus \bigoplus_{j<-1} \mathscr{N}^{j}$ and $w$ arbitrary irreducible and admissible, define $\varphi_{z \times w}: \mathscr{N}^{\cdot} \rightarrow \mathbb{Q}$ by defining for $t$ irreducible $\varphi_{z \times w}(t)=1$ if $t=z \times w$ and 0 otherwise, and extending linearly. A cycle in $\mathscr{N}$ will be called negatively indecomposable if it lies in the kernel of $\varphi_{z \times w}$ for all such choices of $z$ and $w$. Write $\operatorname{NIND}^{p} \subset \mathscr{N}^{p}$ for the subspace of negatively indecomposable cycles. A cycle $Z=\sum n_{i} Z_{i}$ in $\mathscr{N}^{p}\left(Z_{i}\right.$ admissible and irreducible of codimension $r_{i}$ in $X^{2 r_{i}-p}, n_{i} \neq 0$ ) lies in NIND ${ }^{p}$ if and only if no $Z_{i}$ admits a decomposition $Z_{i}=Z_{i 1} \times Z_{i 2}$ where $Z_{i j} \subset X^{2 r_{i j}-p_{j}}$ has codimension $r_{i j}$ with $r_{i 1}+r_{i 2}=r_{i}, p_{1}+p_{2}=p$ such that one of the $p_{j} \leq 0$, and if $p_{j}=0$ with $p_{1}+p_{2}>0$ then $r_{i j}>0$.

Lemma (1.5). (i) $\mathscr{N}^{*}=\mathrm{NIND}^{\circ} \oplus J^{*}$. (ii) NIND ${ }^{\circ}$ is closed under products. It forms a graded subalgebra of $\mathscr{N}^{\cdot}$ (not stable under $\partial$ ).

Proof. (i) seems clear. For (ii), note an alternating cycle is negatively indecomposable if and only if it has no component of the form $z \times w$ with $z$ irreducible of codimension $r \geq 0$ on $X^{2 r-j}$ for $j \leq 0$, the case $r=j=0$ being excluded. Suppose $t_{1}, t_{2}$ are irreducible and the alt $\left(t_{p}\right)$ are negatively indecomposable, with $t_{p}$ codimension $r(p)$ on $X^{2 r(p)-j(p)}$ with $j(p)>0$. Let $S=\left\{1, \ldots, \sum_{p} 2 r(p)-j(p)\right\}$ so $t_{1} \times t_{2} \subset X^{S}$. Let $S=S_{1} \amalg S_{2}$ be the corresponding partition, so $t_{i}$ becomes a cycle on $X^{S_{i}}$. Let $S=T_{1} \amalg T_{2}$ be another partition with $\operatorname{pr}_{k}: X^{S} \rightarrow X^{T_{k}}$. Suppose given $r \geq 0$ and $j \leq 0$ not both 0 such that $\# T_{1}=2 r-j$, and suppose given $z$ a cycle of codimension $r$ on $X^{T_{1}}$. We must rule out an equation $t_{1} \times t_{2}=\operatorname{pr}_{1}^{*}(z) \cdot \operatorname{pr}_{2}^{*}(w)$ for some cycle $w$ on $X^{T_{2}}$. Let $S_{p q}=S_{p} \cap T_{q}$ be the refined partition and let $\operatorname{pr}_{p q}: X^{S} \rightarrow X^{S_{p q}}$. Lemma 4 implies that such an equation comes from a refined product decomposition $t_{1} \times t_{2}=\prod \operatorname{pr}_{p q}^{*}\left(z_{p q}\right)$ where $t_{p}=\operatorname{pr}_{p 1}^{*}\left(z_{p 1}\right) \cdot \operatorname{pr}_{p 2}^{*}\left(z_{p 2}\right)$ and $z=z_{11} \cdot z_{21}$. Let $\# S_{p q}=2 r(p, q)-j(p, q)$ where $z_{p q}$ has codimension $r(p, q)$ on $X^{S_{p q}}$. We have $r=r(1,1)+r(2,1)$ and $0 \geq j=j(1,1)+j(2,1)$. Thus either one 
at least of the $j(p, 1)$ is $<0$, in which case the corresponding $t_{p}$ is not negatively indecomposable (a contradiction), or both the $j(p, 1)=0$. But in the latter case, for $t_{p}$ to be negatively indecomposable, we must have $r(p, 1)=0$ as well so $r=j=0$ and again there is a contradiction. This proves Lemma (1.5). Q.E.D.

Lemma (1.6). We have

$$
\begin{aligned}
& \bigwedge^{2} \mathrm{NIND}^{1} \hookrightarrow \mathrm{NIND}^{2} ; \\
& \left(\mathrm{NIND}^{2} / \bigwedge^{2} \mathrm{NIND}^{1}\right) \otimes \mathrm{NIND}^{1} \hookrightarrow \mathrm{NIND}^{3} / \bigwedge^{3} \mathrm{NIND}^{1} .
\end{aligned}
$$

Proof. Let $Z$ be an irreducible component of a cycle in NIND $^{p}$. Then $Z$ is geometrically irreducible. Suppose $Z \subset X^{2 r-p}$ has codimension $r$. If $Z$ admits a nontrivial decomposition $Z=Z_{1} \times Z_{2}$ with $Z_{i}$ of codimension $r_{i}$ in $X^{2 r_{i}-p_{i}}$ then both $p_{i}>0$. If $Z=Z_{1} \times Z_{2}=Z_{3} \times Z_{4}$ are two distinct such decompositions with $Z_{i}$ of codimension $r_{i}$ on $X^{2 r_{i}-p_{i}}$, then by Lemma (1.4) there is a common refinement

$$
Z=Z_{13} \times Z_{23} \times Z_{14} \times Z_{24}
$$

Suppose $Z_{i j}$ has codimension $r_{i j}$ on $X^{2 r_{i j}-p_{i j}}$. We have $\sum r_{i j}=r$ and $\sum p_{i j}=$ $p$. By negative indecomposability, $p_{i j} \geq 0$ and $p_{i j}=0$ if and only if $r_{i j}=0$, in which case the factor $Z_{i j}$ is trivial. Assuming the decompositions $Z_{1} \times Z_{2}$ and $Z_{3} \times Z_{4}$ distinct, we can have $p_{i j}=0$ for at most one pair $(i, j)$. If $p=2$, this is a contradiction so there is at most one decomposition. The inclusion $\bigwedge^{2} \mathrm{NIND}^{1} \hookrightarrow \mathrm{NIND}^{2}$ follows without difficulty. If $p=3$, one of the $p_{i j}$ is necessarily 0 , which implies that (exchanging $Z_{1}$ and $Z_{2}$ if necessary) that $Z=Z_{1} \times Z_{23} \times Z_{24}$ and $Z_{2}=Z_{23} \times Z_{24}$ with $p_{1}=p_{23}=p_{24}=1$. This implies $\left(\mathrm{NIND}^{2} / \bigwedge^{2} \mathrm{NIND}^{1}\right) \otimes \mathrm{NIND}^{1} \hookrightarrow \mathrm{NIND}^{3} / \bigwedge^{3} \mathrm{NIND}^{1}$ as claimed. Q.E.D.

Recall we have defined $J \subset \mathscr{J} \subset \mathscr{N}$. Define

$$
\mathscr{M}=\left\{x \in(\mathscr{N} / \mathscr{J})^{1} \mid \partial x \in \bigwedge^{2}(\mathscr{N} / \mathscr{J})^{1} \subset(\mathscr{N} / \mathscr{J})^{2}\right\} .
$$

Lemma (1.7). $\partial \mathscr{M} \subset \bigwedge^{2} \mathscr{M} \subset \bigwedge^{2}(\mathscr{N} / \mathscr{J})^{1} \subset(\mathscr{N} / \mathscr{J})^{2}$

Proof. We have NIND $^{\circ} \cong(\mathcal{N} / J)^{\circ}$ as an algebra by Lemma (1.5). Lemma (1.6) implies that $\mathscr{A}=\mathscr{N} / J$ and $\mathscr{J}=\mathscr{J} / J$ satisfy the hypotheses of Lemma (1.3). Lemma (1.7) is a restatement of (1.3.2). Q.E.D.

\section{Properties of}

Let $F$ be a field, and let $\mathscr{M}$ be associated to $F$ as in $\S 1 . \mathscr{M}$ is a subquotient of $\mathscr{N}^{1}=\bigoplus_{r} \mathscr{V}^{r}(2 r-1)$ and is graded by codimension of cycle: $\mathscr{M}=\bigoplus_{r \geq 1} \mathscr{M}_{r}$; $\mathscr{M}_{r}$ a subquotient of $\mathscr{V}^{r}(2 r-1)$. We have

$$
\partial: \mathscr{M}_{r} \rightarrow\left[\bigwedge^{2}\left(\bigoplus_{1 \leq s \leq r-1} \mathscr{M}_{s}\right)\right]_{r},
$$


where the final subscript $r$ refers to the obvious grading on $\Lambda^{2}\left(\bigoplus \mathscr{M}_{s}\right)$. For example

$$
\partial\left(\mathscr{M}_{1}\right)=(0) ; \quad \partial: \mathscr{M}_{2} \rightarrow \bigwedge^{2} \mathscr{M}_{1} .
$$

Proposition (2.1). $\mathscr{M}_{1} \cong F^{\times} \otimes \mathbb{Q}$.

Proof. The relevant piece of the boundary map $\partial: \mathscr{N}^{0} \rightarrow \mathscr{N}^{1}$ maps $\mathscr{V}^{1}(2) \rightarrow$ $\mathscr{V}^{1}(1)$. Totaro has shown [11] that writing $\mathscr{Z}^{r}(s)$ for the larger group of integral cycles on $X^{s}$ meeting faces properly but without the conditions of admissibility and alternation $\left(\right.$ so $\dot{Z}^{r}(s) \otimes \mathbb{Q} \supset \mathscr{V}^{r}(s)$ ), one has

$$
F^{\times} \cong \operatorname{Coker}\left(\mathscr{Z}^{1}(2) \rightarrow \mathscr{Z}^{1}(1)\right) \text {. }
$$

Given $a, b \in F^{\times}$, Totaro writes down a $C_{a, b} \in \mathscr{Z}^{1}(2)$ with

$$
\partial C_{a, b}=(a \cdot b)-(a)-(b) .
$$

It is easy to see this $C_{a, b}$ is admissible. Since $\mathscr{V}^{1}(1) \cong \mathbb{Z}\left[F^{\times}-\{1\}\right]$, it is straightforward from Lemma (1.1) to show $F^{\times} \otimes \mathbb{Q} \cong \operatorname{Coker}\left(\mathscr{V}^{1}(2) \rightarrow \mathscr{V}^{1}(1)\right)$. One checks easily that $J \cap \mathscr{V}^{1}(1)=(0)$ and $\mathscr{J} \cap \mathscr{V}^{1}(1)=\partial\left(\mathscr{V}^{1}(2)\right)$, proving the lemma.

Proposition (2.2). $\operatorname{Coker}\left(\partial: \mathscr{M}_{2} \rightarrow \bigwedge^{2} \mathscr{M}_{1}\right) \cong K_{2}(F) \otimes \mathbb{Q}$. (Milnor $\left.K_{2}\right)$

Proof. It follows from admissibility that

$$
\partial\left(\mathscr{V}^{2}(3)\right) \subset \text { Image }\left(\bigwedge^{2} \mathscr{V}^{1}(1) \rightarrow \mathscr{V}^{2}(2)\right) .
$$

This implies $\mathscr{V}^{2}(3) \rightarrow \mathscr{M}_{2}$. Totaro [op. cit.] proved that

$$
\operatorname{coker}\left(\mathscr{Z}^{2}(3) \rightarrow \mathscr{Z}^{2}(2)\right) \cong K_{2}(F) \text {. }
$$

(This result was first proved by Suslin, working in simplicial rather than cubical coordinates.) He noted that for $a \in F^{\times}-\{1\}$, the cycle

$$
V_{2}(a) \stackrel{\text { def }}{=}\left\{\left(t, 1-t, 1-a t^{-1}\right) \mid t \in \mathbb{P}^{1}\right\} \cap X^{3} \subset X^{3}
$$

satisfies $\partial V_{2}(a)=(a, 1-a) \in \mathscr{Z}^{2}(2)$. Clearly $V_{2}(a)$ is admissible, and the proof of the proposition follows again by Lemma (1.1).

\section{The POLYLOG LIE ALGEBRA}

The purpose of this section is to show the polylog mixed Hodge structures defined in [3] lift to motives in our sense. That is, we will define a graded subco-Lie algebra $\mathscr{M}_{\log } \subset \mathscr{M}$ (or what is the same thing, a quotient $\mathscr{L} \rightarrow \mathscr{L}_{\log }$ ) together with representations associated to $N \geq 1$ and $z \in F^{\times}-\{1\}, M_{N, z} \in$ $\mathscr{M}_{\log } \otimes \operatorname{End}\left(\mathbb{Q}^{[0, N]}\right)$. Our construction is purely algebraic and does not even require $\operatorname{char}(F)=0$. When $F=\mathbb{C}$, it seems likely that further development of the theory will yield a homomorphism $\mathscr{L}_{\mathrm{TMH}} \rightarrow \mathscr{L} \quad\left(\mathscr{L}_{\mathrm{TMH}}=\right.$ Lie algebra 
associated to the Tannakian category of Tate mixed Hodge structures) in such a way that the composition

$$
\mathscr{L}_{\mathrm{TMH}} \rightarrow \mathscr{L} \rightarrow \mathscr{L}_{\log } \rightarrow \operatorname{End}\left(\mathbb{Q}^{[0, N]}\right)
$$

is the mixed Hodge structure defined by Deligne.

Define a map

$$
\rho_{r}: \mathbb{Z}[F-\{0,1\}] \rightarrow \mathscr{M}_{r}
$$

as follows. Take

$$
\rho_{1}(a)=(1-a) \in \mathscr{M}_{1} \cong F^{*} \otimes \mathbb{Q}
$$

For $r \geq 2$, let $T_{0}, \ldots, T_{r-1}$ be homogeneous coordinates on $\mathbb{P}^{r-1}$. For $a \in$ $F-\{0,1\}$, let $V_{r}(a) \subset X^{2 r-1}$ be the subvariety described parametrically by

$$
\begin{array}{r}
V_{r}(a)=\left\{\left(a T_{r-2} / T_{r-1}, \ldots, a T_{0} / T_{r-1},\left(T_{0}-T_{1}\right) / T_{0},\left(T_{1}-T_{2}\right) / T_{1},\right.\right. \\
\left.\left.\ldots,\left(T_{r-2}-T_{r-1}\right) / T_{r-2},\left(T_{r-1}-a T_{0}\right) / T_{r-1}\right)\right\} .
\end{array}
$$

Define

$$
\rho_{r}(a)=\operatorname{alt}\left(V_{r}(a)\right)
$$

For a more careful description of $V_{r}(a)$, let $U_{i 0}, U_{i 1}$ be homogeneous coordinates on the $i$ th copy of $\mathbb{P}^{1}$, and write

$$
u_{i}=U_{i 1} /\left(U_{i 1}-U_{i 0}\right) \text {, }
$$

so $X^{2 r-1}=\operatorname{Spec}\left(F\left[u_{1}, \ldots, u_{2 r-1}\right]\right)$. One has

$$
\begin{aligned}
& u_{1}=T_{r-1} /\left(T_{r-1}-a T_{r-2}\right), \ldots, u_{r-1}=T_{r-1} /\left(T_{r-1}-a T_{0}\right), \\
& u_{r}=T_{0} / T_{1}, \ldots, u_{2 r-2}=T_{r-2} / T_{r-1}, u_{2 r-1}=a^{-1} T_{r-1} / T_{0} .
\end{aligned}
$$

Let $A$ be the algebra over $F$ generated by all the $T_{i} / T_{j}$. One gets

$$
F\left[u_{1}, \ldots, u_{2 r-1}\right] \rightarrow A\left[\left(1-a T_{0} / T_{r-1}\right)^{-1}, \ldots,\left(1-a T_{r-2} / T_{r-1}\right)^{-1}\right],
$$

and hence a closed immersion from the indicated open affine subscheme of $\mathbb{P}^{r-1}$ into $X^{2 r-1}$.

It is now straightforward to show $V_{r}(a)$ is admissible, and to compute $\partial\left(\rho_{r}(a)\right)$. The result is as indicated in (3.5). Most of the boundary terms die. Setting one coordinate $U_{i 0} / U_{i 1}=0, \infty$ either makes another coordinate $=1$ or makes two coordinates equal, so the resulting cycle dies when taking alt. One verifies for $r \geq 2$

$$
\partial \rho_{r}(a)=-(a) \cdot \rho_{r-1}(a)=\rho_{r-1}(a) \cdot(a) .
$$

In particular, $\partial \rho_{r}(a)$ is decomposable, so $\rho_{r}(a) \in \mathscr{M}_{r}$.

Define

$$
\mathscr{M}_{\log , r}=\operatorname{Image}\left(\rho_{r}\right)
$$


It follows that $\mathscr{M}_{\log } \subset \mathscr{M}$ is a graded sub-co-Lie algebra, with dual graded pro-Lie algebra $\mathscr{L}_{\log }^{\log }$ a quotient of $\mathscr{L}$.

To write down the $N$-polylog motif, $M_{N, z}$, associated to $z \in F^{\times}-\{1\}$, one may simply copy from [3]. Define

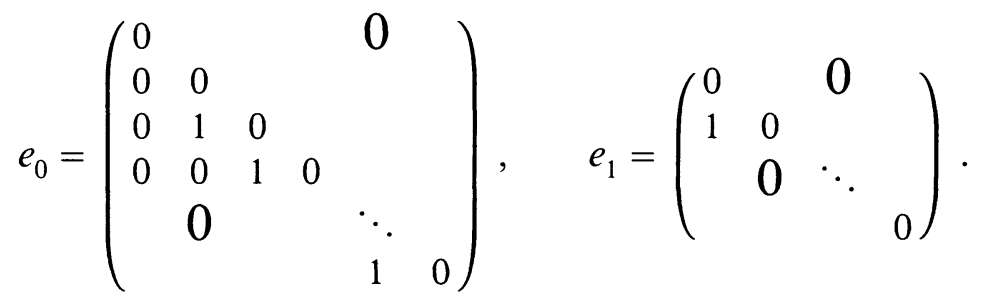

Then $M_{N, z} \in \mathscr{M}_{\log } \otimes \operatorname{End}\left(\mathbb{Q}^{[0, N]}\right)$. Taking

$$
M_{N, z}=-(z) \otimes e_{0}-\sum_{r=1}^{N} \rho_{r}(z) \otimes \operatorname{ad}\left(e_{0}\right)^{r-1}\left(e_{1}\right)
$$

yields

$$
\partial M_{N, z}=\left[M_{N, z}, M_{N, z}\right]
$$

so the resulting map $\mathscr{L}_{\log } \rightarrow \operatorname{End}\left(\mathbb{Q}^{[0, N]}\right)$ is a representation.

Remark (3.8). It follows from (3.5), Lemma (1.7), and induction that when $F^{\times}$ is not a torsion group, $\mathscr{M}_{r} \neq(0)$ for any $r$. In particular, our theory is not trivial!

\section{RELATION WITH MiNimal MODELS}

Let $V$ be a smooth affine variety defined over $\mathbb{C}$. One knows that the algebraic de Rham complex $\Omega_{V}^{*}$ calculates the singular cohomology $H^{*}(V(\mathbb{C}), \mathbb{C})$. Writing $\mathscr{E}_{V}^{\cdot}$ for the complex of $\mathbb{C}$-valued $C^{\infty}$-forms, one has that the natural map $\lambda: \Omega_{V} \rightarrow \mathscr{E}_{V}^{*}$ is a quasi-isomorphism. Recall that an $n$-minimal model $\mathscr{Q}^{*}$ of a differential graded algebra $\mathscr{A}^{\cdot}\left(\mathscr{A}^{i}=(0)\right.$ for $\left.i<0\right)$ is a DGA $\mathscr{Q}^{\cdot}$ satisfying certain minimality properties independent of $\mathscr{A}$ (we will recall the construction of the 1-minimal model, which is the only one we need, below) together with a map $\imath: \mathscr{Q}^{*} \rightarrow \mathscr{A}^{\cdot}$ such that $l^{r}: H^{r}(\mathscr{Q}) \rightarrow H^{r}(\mathscr{A})$ is an isomorphism for $r \leq n$ and injective for $r=n+1$. Hence, if $(\mathscr{Q}, l)$ is an $n$-minimal model for $\Omega_{V}^{*}$, then $(\mathscr{Q}, \lambda \circ l)$ is an $n$-minimal model for $\mathscr{E}_{V}$.

The 1-minimal model for $\Omega_{V}$ is an exterior algebra $\Lambda^{\circ} \mathscr{Q}$ on a $\mathbb{C}$-vector space $\mathscr{Q}$ in degree 1. Further, $\mathscr{Q}=\bigcup \mathscr{Q}(i), i \geq 1$, with $\mathscr{Q}(i-1) \subset \mathscr{Q}(i)$ and the differential

$$
\partial: \mathscr{Q}(i) \rightarrow \bigwedge^{2} \mathscr{Q}(i-1) .
$$

In particular, the differential on $\mathscr{Q}(1)$ is zero. $\mathscr{Q}(i)$ is dual to the Lie algebra associated to the nilpotent group $\pi_{1}(V) / \pi_{1}^{(i+1)}$ and $\partial: \mathscr{Q}(i) \rightarrow \bigwedge^{2} \mathscr{Q}(i)$ is dual to the Lie bracket. (This identification depends on the choice of a basepoint.) 
Let $\Omega^{\circ}=\Omega_{\mathbb{C}(V) / \mathbb{C}}$ be the de Rham algebra of the function field of $V$, and write $\mathscr{M}_{\text {top }}=\bigcup \mathscr{M}_{\text {top }}(i)$ for the degree 1 part of the 1 minimal model of $\Omega^{\circ}$.

We begin with some comments about the construction of $\mathscr{M}_{\text {top }} \cdot \mathscr{M}_{\text {top }}(1) \subset$ $\Omega_{\text {closed }}^{1}$ must be chosen so $\mathscr{M}_{\text {top }}(1) \cong H^{1}\left(\Omega^{*}\right)$. The map dlog: $\mathbb{C}(V)^{\times} / \mathbb{C}^{\times} \rightarrow$ $\Omega_{\text {closed }}^{1}$ is defined by $\operatorname{dlog}(f)=d f / f$. By composing with the residue, one sees immediately that the resulting map $\mathbb{C} \otimes\left(\mathbb{C}(V)^{\times} / \mathbb{C}^{\times}\right) \rightarrow H^{1}\left(\Omega^{*}\right)$ is injective. Thus we may (and do) assume

$$
\mathbb{C} \otimes \operatorname{dlog}\left(\mathbb{C}(V)^{\times} / \mathbb{C}^{\times}\right) \subset \mathscr{M}_{\text {top }}(1) .
$$

Assume

$$
\begin{aligned}
& \mathscr{M}_{\text {top }}(i-1) ; \quad \partial: \mathscr{M}_{\text {top }}(i-1) \rightarrow \bigwedge^{2} \mathscr{M}_{\text {top }}(i-2) \subset \bigwedge^{2} \mathscr{\mathscr { M }}_{\text {top }}(i-1) ; \quad \text { and } \\
& \alpha(i-1): \mathscr{M}_{\text {top }}(i-1) \rightarrow \Omega^{1}
\end{aligned}
$$

defined so

$$
\bigwedge^{\cdot} \alpha(i-1): \bigwedge^{\cdot} \mathscr{M}_{\mathrm{top}}(i-1) \rightarrow \Omega^{\cdot}
$$

is compatible with differentials and is an isomorphism on $H^{1}$. Take

$$
\mathscr{W}_{i}=\operatorname{Ker}\left(H^{2}\left(\bigwedge^{\cdot} \mathscr{M}_{\text {top }}(i-1)\right) \rightarrow H^{2}\left(\Omega^{*}\right) .\right.
$$

Define

$$
\begin{aligned}
& Z^{2}\left(\bigwedge^{\cdot} \mathscr{M}_{\mathrm{top}}(i-1), \Omega^{\cdot}\right) \\
& \quad=\left\{(m, \omega) \in \bigwedge^{2} \mathscr{M}_{\mathrm{top}}(i-1) \times \Omega^{1} \mid \partial m=0 ;\left(\bigwedge^{2} \alpha(i-1)\right)(m)=d \omega\right\}
\end{aligned}
$$

$\left(Z^{2}=2\right.$-cocyles for the mapping cone of $\alpha(i-1)$.) Define

$$
\begin{aligned}
X(i-1) & \stackrel{\text { def }}{=}\left\{m \in \bigwedge^{2} \mathscr{M}_{\mathrm{top}}(i-1) \mid \partial m=0=\bigwedge^{2} \alpha(i-1)(m)\right\} \\
& \subset Z^{2}\left(\bigwedge^{\cdot} \mathscr{M}_{\mathrm{top}}(i-1), \Omega^{\circ}\right) .
\end{aligned}
$$

Write

$$
p: Z^{2} \rightarrow \bigwedge^{2} \mathscr{M}_{\text {top }}(i-1) \quad\left(\text { resp. } q: Z^{2} \rightarrow \Omega^{1}\right)
$$

for the projections. Let

$$
s: \mathscr{W}_{i} \hookrightarrow Z^{2}\left(\bigwedge^{\cdot} \mathscr{M}_{\text {top }}(i-1), \Omega^{*}\right)
$$

be a chosen splitting of the natural surjection $t: Z^{2} \rightarrow \mathscr{W}_{i}$. Write

$$
\mathscr{X}_{i}=t(X(i-1)) \subset \mathscr{W}_{i} .
$$

We can assume

$$
s\left(\mathscr{X}_{i}\right) \subset X(i-1)
$$


Take $\mathscr{M}_{\text {top }}(i)=\mathscr{M}_{\text {top }}(i-1) \oplus \mathscr{W}_{i}$. Define

$$
\begin{gathered}
\partial: \mathscr{M}_{\text {top }}(i) \rightarrow \bigwedge^{2} \mathscr{M}_{\text {top }}(i-1) \subset \bigwedge^{2} \mathscr{M}_{\text {top }}(i), \\
\partial \mid \mathscr{M}_{\text {top }}(i-1)=\text { inductively defined map; } \quad \partial \mid \mathscr{W}_{i}=p \circ s .
\end{gathered}
$$

Define

$$
\begin{gathered}
\alpha(i): \mathscr{M}_{\text {top }}(i) \rightarrow \Omega^{1} \\
\alpha(i)\left|\mathscr{M}_{\text {top }}(i-1)=\alpha(i-1) ; \quad \alpha(i)\right| \mathscr{W}_{i}=q \circ s .
\end{gathered}
$$

One checks without difficulty that $\dot{\mathscr{M}}_{\text {top }}(i), \partial$, and $\alpha(i)$ satisfy the required hypotheses. Note that (4.7) implies

$$
\alpha(i)\left(\mathscr{X}_{i}\right)=(0) .
$$

In the next two sections, we will define a map of co-Lie algebras

$$
\varphi: \mathscr{M} \rightarrow \mathscr{M}_{\text {top }}
$$

where $\mathscr{M}$ is the co-Lie algebra defined in $\S 1$. This could be done directly, but it is more informative to construct $\varphi$ as a composition

$$
\mathscr{M} \stackrel{\gamma}{\rightarrow} \mathscr{P} \stackrel{\beta}{\rightarrow} \mathscr{M}_{\text {top }}
$$

Here $\mathscr{P}=\mathscr{P}_{1} \oplus \mathscr{P}_{2} \oplus \cdots$ is a graded $\mathbb{Q}$-co-Lie algebra and $\gamma$ is a morphism of graded co-Lie algebras. $\mathscr{P}$ depends only on the vector space

$$
\mathscr{P}_{1} \stackrel{\text { def }}{=}\left(\mathbb{C}(V)^{\times} / \mathbb{C}^{\times}\right) \otimes \mathbb{Q}
$$

and

The morphism

$$
\mathscr{P}_{2} \stackrel{\text { def }}{=} \operatorname{Ker}\left(\operatorname{dlog} \wedge \operatorname{dlog}: \bigwedge^{2}\left(\mathbb{C}(V)^{\times} / \mathbb{C}^{\times}\right) \otimes \mathbb{Q} \rightarrow \Omega^{2}\right)
$$

$$
\gamma_{1}: \mathscr{M}_{1}=\mathbb{C}(V)^{\times} \otimes \mathbb{Q} \rightarrow \mathscr{P}_{1} \stackrel{\text { def }}{=}\left(\mathbb{C}(V)^{\times} / \mathbb{C}^{\times}\right) \otimes \mathbb{Q}
$$

is the obvious one. Details of the construction of $\mathscr{P}$ and $\gamma$ are given in $\S 5$.

$\mathscr{P}$ is filtered by sub-co-Lie algebras $\mathscr{P}(n)=\mathscr{P}_{1} \oplus \cdots \oplus \mathscr{P}_{n}$, and $\beta(\mathscr{P}(n)) \subset$ $\mathscr{M}_{\text {top }}(n)$. The map $\beta$ is constructed in $\S 6$.

\section{Construction of CERTAin GRAdEd Co-Lie Algebras}

Let $\mathscr{P}_{1}$ be a vector space, and let $\mathscr{P}_{2} \subset \bigwedge^{2} \mathscr{P}_{1}$ be a given subspace. We will define inductively vector spaces $\mathscr{P}_{n}$ and boundary maps

$$
\partial=\partial(n): \mathscr{P}(n) \stackrel{\text { def }}{=} \bigoplus_{1 \leq j \leq n} \mathscr{P}_{n} \rightarrow \bigwedge^{2} \mathscr{P}(n-1) \subset \bigwedge^{2} \mathscr{P}(n)
$$

Extending $\partial$ in the usual way to an endomorphism of $\wedge^{\circ} \mathscr{P}(n)$ we will have $\partial \circ \partial=0$, and writing $\mathscr{P}=\bigoplus_{1 \leq j<\infty} \mathscr{P}_{n}$

$$
H^{1}\left(\bigwedge^{\cdot} \mathscr{P}\right)=\mathscr{P}_{1} ; \quad H^{2}\left(\bigwedge^{\cdot} \mathscr{P}\right)=\left(\bigwedge^{2} \mathscr{P}_{1}\right) / \mathscr{P}_{2} .
$$

The dual $\mathscr{R}$ of $\mathscr{P}$ will be a (pro) Lie algebra with these cohomology groups. 
Inductive hypothesis (5.3). Let $n \geq 3$, and supppose $\mathscr{P}_{1}, \ldots, \mathscr{P}_{n-1}$ defined along with

$$
\partial(m): \mathscr{P}(m) \rightarrow \bigwedge^{2} \mathscr{P}(m-1) \subset \bigwedge^{2} \mathscr{P}(m) ; \quad m<n
$$

We assume the following conditions satisfied for $m<n$ :

(i) $\partial(m) \mid \mathscr{P}(m-1)=\partial(m-1)$.

(ii) $\operatorname{Ker}\left(\partial(m): \mathscr{P}(m) \rightarrow \bigwedge^{2} \mathscr{P}(m)\right)=\mathscr{P}(1)$.

(iii) $\partial(m)^{2}=0$.

(iv) $\partial(m)$ is homogeneous of degree 0 for the grading on $\wedge^{\cdot} \mathscr{P}(m)$, which assigns grade $\sum r_{i}$ to an element $q_{1} \wedge \cdots \wedge q_{p}$ with $q_{i} \in \mathscr{P}_{r_{i}}$.

(v) For $3 \leq m<n$,

$\mathscr{P}_{m}=\operatorname{Ker}\left[\bigwedge^{2} \mathscr{P}(m-1) \rightarrow \bigwedge^{3} \mathscr{P}(m-1)\right] /\left[\bigwedge^{2} \mathscr{P}(1)+\partial \mathscr{P}(m-1)\right]$.

(vi) For $3 \leq m<n$,

$$
\mathscr{P}_{m} \cong \operatorname{Ker}\left[\bigwedge^{2} \mathscr{P}(m-1) \rightarrow \bigwedge^{3} \mathscr{P}(m-1)\right]_{m},
$$

where the subscript $m$ refers to the piece of graded degree $m$. The boundary map

$$
\partial(m) \mid \mathscr{P}_{m}: \mathscr{P}_{m} \rightarrow \bigwedge^{2} \mathscr{P}(m-1)
$$

is induced from this isomorphism.

Note $\partial(1)=0$, and

$$
\partial(2): \mathscr{P}_{1} \oplus \mathscr{P}_{2} \rightarrow \mathscr{P}_{2} \subset \bigwedge^{2} \mathscr{P}_{1}
$$

satisfy conditions (5.3).

Inductive step (5.4). Suppose $n \geq 3$, and these conditions hold for $m<n$. Define

$$
\mathscr{P}_{n}=\operatorname{Ker}\left[\bigwedge^{2} \mathscr{P}(n-1) \rightarrow \bigwedge^{3} \mathscr{P}(n-1)\right] /\left[\bigwedge^{2} \mathscr{P}(1)+\partial \mathscr{P}(n-1)\right] .
$$

Lemma (5.4.2). Assume $n \geq 3$. Then

Proof. We have

$$
\mathscr{P}_{n} \cong \operatorname{Ker}\left[\bigwedge^{2} \mathscr{P}(n-1) \rightarrow \bigwedge^{3} \mathscr{P}(n-1)\right]_{n}
$$

$$
\begin{gathered}
\mathscr{P}(n-1)=\mathscr{P}(n-2) \oplus \mathscr{P}_{n-1} ; \\
\bigwedge^{2} \mathscr{P}(n-1)=\left(\bigwedge^{2} \mathscr{P}(n-2)\right) \oplus\left(\mathscr{P}(n-2) \otimes \mathscr{P}_{n-1}\right) \oplus \bigwedge^{2} \mathscr{P}_{n-1}
\end{gathered}
$$


The boundary $\partial(n-1) \mid \bigwedge^{2} \mathscr{P}_{n-1}$ is the composition

$$
\begin{aligned}
& \bigwedge^{2} \mathscr{P}_{n-1} \rightarrow \stackrel{2}{\bigotimes} \mathscr{P}_{n-1} \rightarrow\left(\bigwedge^{2} \mathscr{P}(n-2)\right) \otimes \mathscr{P}_{n-1}, \\
& x \wedge y \mapsto x \otimes y-y \otimes x ; \quad z \mapsto(\partial(n-1) \otimes \mathrm{id})(z) .
\end{aligned}
$$

Both these arrows are injective. Since $\partial(n-1)$ is homogeneous, we get

$$
\begin{aligned}
\operatorname{Ker} & {\left[\bigwedge^{2} \mathscr{P}(n-1) \rightarrow \bigwedge^{3} \mathscr{P}(n-1)\right] } \\
& \cong \operatorname{Ker}\left[\bigwedge^{2} \mathscr{P}(n-2) \oplus\left(\mathscr{P}(n-2) \otimes \mathscr{P}_{n-1}\right) \rightarrow \bigwedge^{3} \mathscr{P}(n-1)\right] .
\end{aligned}
$$

Write an element in this kernel as

$$
b+a ; \quad b \in \bigwedge^{2} \mathscr{P}(n-2) ; \quad a \in \mathscr{P}(n-2) \otimes \mathscr{P}_{n-1} .
$$

If $a=0$, it follows from (v) and (vi) above applied with $m=n-1$ that

$$
b \in \bigwedge^{2} \mathscr{P}(1)+\partial \mathscr{P}(n-1) \text {. }
$$

The only component of $\partial(b+a)$ involving $\mathscr{P}_{n-1}$ is

$$
(\partial \otimes \mathrm{id})(a) \in \partial \mathscr{P}(n-2) \otimes \mathscr{P}_{n-1} \subset \bigwedge^{3} \mathscr{P}(n-1),
$$

so by (ii)

$$
a \in \mathscr{P}(1) \otimes \mathscr{P}_{n-1}=\mathscr{P}_{1} \otimes \mathscr{P}_{n-1} .
$$

In particular, $a$ is homogeneous of degree $n$, and Lemma (5.4.2) follows.

Remark (5.4.3). For $n \geq 3$, the isomorphism

$$
\begin{aligned}
& \operatorname{Ker} {\left[\bigwedge^{2} \mathscr{P}(n-1) \rightarrow \bigwedge^{3} \mathscr{P}(n-1)\right] /\left[\bigwedge^{2} \mathscr{P}(1)+\partial \mathscr{P}(n-1)\right] } \\
& \cong \operatorname{Ker}\left[\bigwedge^{2} \mathscr{P}(n-1) \rightarrow \bigwedge^{3} \mathscr{P}(n-1)\right]_{n}
\end{aligned}
$$

is given by projection on the component of degree $n$. The inverse is the obvious map. Note the argument in the lemma does not imply

$$
\operatorname{Ker}\left[\bigwedge^{2} \mathscr{P}(n-1) \rightarrow \bigwedge^{3} \mathscr{P}(n-1)\right]_{n} \subset \mathscr{P}_{1} \otimes \mathscr{P}_{n-1} .
$$

We now complete the inductive step (5.4). Define

$$
\begin{aligned}
\mathscr{P}(n)=\mathscr{P}(n-1) \oplus \mathscr{P}_{n} ; \quad \partial(n) \mid \mathscr{P}(n-1)=\partial(n-1) ; \\
\partial(n) \mid \mathscr{P}_{n}: \mathscr{P}_{n} \cong \operatorname{Ker}\left[\bigwedge^{2} \mathscr{P}(n-1) \rightarrow \bigwedge^{3} \mathscr{P}(n-1)\right]_{n} \\
\subset \bigwedge^{2} \mathscr{P}(n-1) \subset \bigwedge^{2} \mathscr{P}(n) .
\end{aligned}
$$

One checks easily that (i)-(vi) hold with $m=n$. This completes the inductive construction. 
Examples (5.5). (1) The argument of Lemma (5.4.2) does imply that the projection

$$
\mathscr{P}_{n}=\operatorname{Ker}\left[\bigwedge^{2} \mathscr{P}(n-1) \rightarrow \bigwedge^{3} \mathscr{P}(n-1)\right]_{n} \rightarrow \mathscr{P}_{1} \otimes \mathscr{P}_{n-1}
$$

is injective. If $\mathscr{P}_{p}=0$ therefore, $\mathscr{P}_{n}=0$ for $n \geq p$. In particular, if $\mathscr{P}_{2}=0$, we get $\mathscr{P}=\mathscr{P}_{1}, \partial=0$, and the corresponding Lie algebra is abelian.

(2) We show in (5.7) below that when $\mathscr{P}_{2}=\bigwedge^{2} \mathscr{P}_{1}$, the Lie algebra $\mathscr{R}$ is the free Lie algebra on $\mathscr{P}_{1}$.

(3) The construction is functorial; a map $\mathscr{P}_{1} \rightarrow \mathscr{P}_{1}^{\prime}$ inducting $\mathscr{P}_{2} \rightarrow \mathscr{P}_{2}^{\prime}$ gives a map $\mathscr{P}\left(\mathscr{P}_{1}, \mathscr{P}_{2}\right) \rightarrow \mathscr{P}\left(\mathscr{P}_{1}^{\prime}, \mathscr{P}_{2}^{\prime}\right)$ and hence $\mathscr{R}\left(\mathscr{P}_{1}^{\prime}, \mathscr{P}_{2}^{\prime}\right) \rightarrow \mathscr{R}\left(\mathscr{P}_{1}, \mathscr{P}_{2}\right)$.

Questions (5.6). What is the structure of the quotient co-Lie algebra $\mathscr{P}_{2} \oplus$ $\mathscr{P}_{3} \oplus \cdots$ ?

What can one say when $\mathscr{P}_{2}$ is generated by decomposable tensors $a \wedge b$ ?

Proposition (5.7). $\mathscr{R}\left(\mathscr{P}_{1}, \wedge^{2} \mathscr{P}_{1}\right)$ is the free (pro)-Lie algebra on the (pro)-vector space $\mathscr{P}_{1}^{\vee}$.

Proof. Let $\mathscr{Q}$ be a co-Lie algebra. Define $\mathscr{Q}(1)=\operatorname{Ker}\left(\partial: \mathscr{Q} \rightarrow \bigwedge^{2} \mathscr{Q}\right)$. Suppose given $\rho(1): \mathscr{Q}(1) \rightarrow \mathscr{P}(1)=\mathscr{P}_{1}$. Assume inductively that sub-co-Lie algebras $\mathscr{Q}(m) \subset \mathscr{Q}$ are defined for $m<n$, and define

$$
\begin{aligned}
\left(\bigwedge^{2} \mathscr{Q}\right)(n) & =\left(\bigwedge^{2} \mathscr{Q}(n-1)\right)(n) \\
& =\text { Image }\left[\sum_{p+r=n} \mathscr{Q}(p) \otimes \mathscr{Q}(r) \rightarrow \bigwedge^{2} \mathscr{Q}(n-1)\right] .
\end{aligned}
$$

Define

$$
\mathscr{Q}(n)=\partial^{-1}\left[\left(\bigwedge^{2} \mathscr{Q}\right)(n)\right] .
$$

Assume $\mathscr{Q}=\bigcup \mathscr{Q}(n)$, and suppose inductively that $\rho(n-1): \mathscr{Q}(n-1) \rightarrow$ $\mathscr{P}(n-1)$, a homomorphism of filtered co-Lie algebras is defined. Consider the diagram

$$
\begin{aligned}
& 0 \rightarrow \mathscr{Q}(1) \rightarrow \mathscr{Q}(n) \rightarrow\left(\Lambda^{2} \mathscr{Q}(n-1)\right)(n) \rightarrow\left(\Lambda^{3} \mathscr{Q}(n-1)\right)(n) \\
& \downarrow \rho(1) \quad \downarrow ? \quad \downarrow \Lambda^{2} \rho(n-1) \quad \downarrow \Lambda^{3} \rho(n-1) \\
& 0 \rightarrow \mathscr{P}(1) \rightarrow \mathscr{P}(n) \rightarrow\left(\Lambda^{2} \mathscr{P}(n-1)\right)(n) \rightarrow\left(\Lambda^{3} \mathscr{P}(n-1)\right)(n) .
\end{aligned}
$$

The top line is a complex that is exact at $\mathscr{Q}(n)$. The bottom line is exact by (5.2), and the arrows are already defined except the one labeled ?. It follows easily that $\rho(n)=$ ? can be defined to make the diagram commute. Finally, $\rho=\underset{\lim }{\longrightarrow} \rho(n)$ is dual to a homomorphism of Lie algebras, so $\mathscr{P}^{\vee}$ is free. Q.E.D.

Proposition (5.8). With reference to (5.5)(3), consider the case $\mathscr{P}_{1}=\mathscr{P}_{1}^{\prime}, \mathscr{P}_{2} \subset$ $\mathscr{P}_{2}^{\prime}$. We have

$$
\mathscr{P}_{2}^{\prime \vee}=\left[\mathscr{R}\left(\mathscr{P}_{1}, \mathscr{P}_{2}^{\prime}\right)_{-1}, \mathscr{R}\left(\mathscr{P}_{1}, \mathscr{P}_{2}^{\prime}\right)_{-1}\right] .
$$


Moreover, $\mathscr{R}\left(\mathscr{P}_{1}, \mathscr{P}_{2}\right) \cong \mathscr{R}\left(\mathscr{P}_{1}, \mathscr{P}_{2}^{\prime}\right) / I$, where $I$ is the ideal generated by $\left(\mathscr{P}_{2}^{\prime} / \mathscr{P}_{2}\right)^{\vee}$.

Proof. The first statement is clear. For the second, consider the diagram for $n \geq 3$.

$$
\begin{aligned}
& {\left[\bigwedge^{3} \mathscr{P}\left(\mathscr{P}_{1}, \mathscr{P}_{2}^{\prime}\right)(n-1)^{\vee}\right]_{-n} \rightarrow\left[\bigwedge^{2} \mathscr{P}\left(\mathscr{P}_{1}, \mathscr{P}_{2}^{\prime}\right)(n-1)^{\vee}\right]_{-n} \rightarrow \mathscr{P}_{n}^{\prime \vee} \rightarrow 0} \\
& {\left[\wedge^{3} \mathscr{P}\left(\mathscr{P}_{1}, \mathscr{P}_{2}\right)(n-1)^{\vee}\right]_{-n} \rightarrow\left[\bigwedge^{2} \mathscr{P}\left(\mathscr{P}_{1}, \mathscr{P}_{2}\right)(n-1)^{\vee}\right]_{-n} \rightarrow \begin{array}{c}
\downarrow b \\
\downarrow
\end{array} \mathscr{\mathscr { P }}_{n}^{\vee} \rightarrow 0}
\end{aligned}
$$

Surjectivity of vertical arrows is verified by induction on $n$, since surjectivity of the two left-hand arrows implies surjectivity on the right. A diagram chase shows $\operatorname{Ker}(a) \rightarrow \operatorname{Ker}(b)$. Again by induction on $n$ we deduce that $\operatorname{Ker}(b)=$ $\left(\mathscr{P}_{2}^{\prime} / \mathscr{P}_{2}\right)^{\vee} \oplus\left[\mathscr{R}\left(\mathscr{P}_{1}, \mathscr{P}_{2}^{\prime}\right),\left(\mathscr{P}_{2}^{\prime} / \mathscr{P}_{2}\right)^{\vee}\right]$. Q.E.D.

Corollary (5.9). $\mathscr{R}\left(\mathscr{P}_{1}, \mathscr{P}_{2}\right) \cong \mathscr{R}\left(\mathscr{P}_{1}, \bigwedge^{2} \mathscr{P}_{1}\right) / I$, where I is the ideal generated by $\left(\bigwedge^{2} \mathscr{P}_{1} / \mathscr{P}_{2}\right)^{\vee} \subset\left[\mathscr{R}\left(\mathscr{P}_{1}, \bigwedge^{2} \mathscr{P}_{1}\right)_{-1}, \mathscr{R}\left(\mathscr{P}_{1}, \bigwedge^{2} \mathscr{P}_{1}\right)_{-1}\right]$.

Finally we construct a graded map

$$
\gamma: \mathscr{M} \rightarrow \mathscr{P}\left(\mathscr{P}_{1}, \mathscr{P}_{2}\right)
$$

with

$$
\mathscr{P}_{1}=\mathbb{C}(V)^{\times} / \mathbb{C}^{\times} \otimes \mathbb{Q} ; \quad \mathscr{P}_{2}=\operatorname{Ker}\left(\bigwedge^{2}\left(\mathbb{C}(V)^{\times} / \mathbb{C}^{\times} \otimes \mathbb{Q}\right) \rightarrow \Omega^{2}\right) .
$$

The map $\gamma_{1}: \mathscr{M}_{1} \rightarrow \mathscr{P}_{1}$ is the obvious one (4.9).

To define $\gamma_{2}: \mathscr{M}_{2} \rightarrow \mathscr{P}_{2}$ consider the diagram

$$
\begin{array}{cc}
\mathscr{M}_{2} \stackrel{\partial}{\rightarrow} \Lambda^{2} \mathscr{M}_{1} \cong \Lambda^{2} \mathbb{C}(V)^{\times} \otimes \mathbb{Q} \rightarrow & K_{2}(\mathbb{C}(V)) \otimes \mathbb{Q} \rightarrow 0 \\
\operatorname{dlog} \wedge \mathrm{dlog} \backslash & \downarrow \\
\Omega^{2}
\end{array}
$$

where the top line is exact by (2.2) and the factorization of $\operatorname{dlog} \wedge$ dlog exists, e.g., because the relations in $K_{2}$ are generated by the Steinberg relation $x \otimes$ $(1-x) \mapsto 0$. In particular the composite

$$
\mathscr{M}_{2} \stackrel{\partial}{\rightarrow} \bigwedge^{2} \mathscr{M}_{1} \rightarrow \bigwedge^{2} \mathscr{P}_{1}
$$

has image $\subset \mathscr{P}_{2}$. Write $\gamma_{2}: \mathscr{M}_{2} \rightarrow \mathscr{P}_{2}$ for this map. The diagram

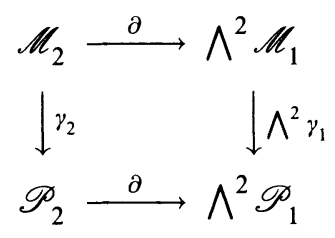

commutes. 
Assume inductively for some $n \geq 3$ that $\gamma_{m}: \mathscr{M}_{m} \rightarrow \mathscr{P}_{m}$ has been defined for $m \leq n-1$. We have

$$
\begin{aligned}
& \mathscr{M}_{n} \stackrel{\partial}{\rightarrow}\left[\Lambda^{2}\left(\mathscr{M}_{1} \oplus \cdots \oplus \mathscr{M}_{n-1}\right)\right]_{n} \stackrel{\partial}{\rightarrow}\left[\Lambda^{3}\left(\mathscr{M}_{1} \oplus \cdots \oplus \mathscr{M}_{n-1}\right)\right]_{n} \\
& \downarrow \Lambda^{2} \gamma \quad \downarrow \Lambda^{3} \gamma \\
& 0 \rightarrow \mathscr{P}_{n} \stackrel{\partial}{\rightarrow}\left[\Lambda^{2}\left(\mathscr{P}_{1} \oplus \cdots \oplus \mathscr{P}_{n-1}\right)\right]_{n} \stackrel{\partial}{\rightarrow}\left[\Lambda^{3}\left(\mathscr{P}_{1} \oplus \cdots \oplus \mathscr{P}_{n-1}\right)\right]_{n}
\end{aligned}
$$

where the bottom row is exact. There is a unique map

making the diagram commute.

$$
\gamma_{n}: \mathscr{M}_{n} \rightarrow \mathscr{P}_{n}
$$

\section{THE MAP $\beta$}

Write $\mathscr{P}=\left(\mathscr{P}_{1}, \mathscr{P}_{2}\right)$ with $\mathscr{P}_{i}$ as in $(5.11)$. Let $\mathscr{P}(n)=\mathscr{P}_{1} \oplus \cdots \oplus \mathscr{P}_{n}$. In this section we define a morphism of graded co-Lie algebras

$$
\beta: \mathscr{P} \rightarrow \mathscr{M}_{\text {top }}
$$

We use the notations of $\S 4$.

$$
\beta(1): \mathscr{P}(1)=\left(\mathbb{C}(V)^{\times} / \mathbb{C}^{\times}\right) \otimes \mathbb{Q} \rightarrow \mathscr{M}_{\text {top }}(1)
$$

is the given inclusion (4.2). Write $\vartheta_{2}$ for the inclusion

$$
\vartheta_{2}: \mathscr{P}_{2} \subset X(1) \subset \bigwedge^{2} \mathscr{M}_{\text {top }}(1)
$$

so there is a natural map

$$
\beta_{2} \stackrel{\text { def }}{=} t \circ \vartheta_{2}: \mathscr{P}_{2} \rightarrow \mathscr{X}_{2} \subset \mathscr{W}_{2} \subset \mathscr{M}_{\text {top }}(2) .
$$

Write

$$
\beta(2)=\beta(1) \oplus \beta_{2}: \mathscr{P}(2)=\mathscr{P}(1) \oplus \mathscr{P}_{2} \rightarrow \mathscr{M}_{\text {top }}(2)=\mathscr{M}_{\text {top }}(1) \oplus \mathscr{W}_{2} .
$$

Since $\partial=0$ on $\mathscr{M}_{\text {top }}(1), t: X(1) \cong \mathscr{X}_{2}$ and $s \circ t=$ id on $X(1)$. It follows that the diagram

$$
\begin{aligned}
& \mathscr{P}(2) \longrightarrow \Lambda^{2} \mathscr{P}(1) \\
& \downarrow \beta(2) \quad \downarrow \wedge^{2} \beta(1) \\
& \mathscr{M}_{\text {top }}(2) \longrightarrow \bigwedge^{2} \mathscr{M}_{\text {top }}(1)
\end{aligned}
$$

commutes.

Let $\dot{n} \geq 3$, and suppose inductively

$$
\beta_{i}: \mathscr{P}_{i} \rightarrow \mathscr{X}_{2} \oplus \cdots \oplus \mathscr{X}_{i} \subset \mathscr{M}_{\text {top }}(i)
$$

has been defined for $2 \leq i \leq n-1$. Consider the commutative diagram defining $\vartheta_{n}^{(0)}$ :

$$
\begin{array}{ll}
\Lambda^{3}\left(\bigoplus_{\substack{i \leq n-1 \\
\uparrow \partial}} \mathscr{P}_{j}\right) \quad \stackrel{\Lambda^{3} \beta}{\longrightarrow} \Lambda^{3} \mathscr{M}_{\text {top }}(n-1) \\
\Lambda^{2}\left(\bigoplus_{i \leq n-1} \mathscr{P}_{i}\right) & \stackrel{\Lambda^{2} \beta}{\longrightarrow} \underbrace{\Lambda^{2} \mathscr{M}_{\text {top }}(n-1)}_{\vartheta_{n}^{(0)}}
\end{array}
$$


One has from this that

$$
\vartheta_{n}^{(0)}\left(\mathscr{P}_{n}\right) \subset \operatorname{Ker}(\partial)
$$

Also since $n \geq 3$, the projection $\bigwedge^{2}\left(\bigoplus_{i \leq n-1} \mathscr{P}_{i}\right) \rightarrow \bigwedge^{2} \mathscr{P}_{1}$ maps $\partial\left(\mathscr{P}_{n}\right)$ to 0 . Since $\beta\left(\mathscr{P}_{i}\right) \subset \operatorname{Ker}(\alpha)$ for $2 \leq i \leq n-1$, we see also that

$$
\vartheta_{n}^{(0)}\left(\mathscr{P}_{n}\right) \subset \operatorname{Ker}\left(\bigwedge^{2} \alpha\right)
$$

whence

$$
\vartheta_{n}^{(0)}\left(\mathscr{P}_{n}\right) \subset X(n-1)
$$

Define

$$
\beta_{n}^{(0)}=t \circ \vartheta_{n}^{(0)}
$$

Unfortunately it is no longer the case that $t \mid X(n-1)$ is injective, so the analogue of the argument showing (6.6) commutative gives only

$$
\vartheta_{n}^{(1)} \stackrel{\text { def }}{=}\left(\bigwedge^{2} \beta\right) \circ \partial-\partial \circ \beta_{n}^{(0)}: \mathscr{P}_{n} \rightarrow \operatorname{Ker}(t \mid X(n-1)) \text {. }
$$

Write

$$
C^{1}\left(\bigwedge^{\cdot} \mathscr{M}_{\text {top }}(n-1), \Omega^{\cdot}\right)=\mathscr{M}_{\text {top }}(n-1) \oplus \mathbb{C}(V)
$$

and define

$$
\begin{gathered}
d: C^{1}\left(\bigwedge^{\cdot} \mathscr{M}_{\mathrm{top}}(n-1), \Omega^{\cdot}\right) \rightarrow Z^{2}\left(\bigwedge^{\cdot} \mathscr{M}_{\mathrm{top}}(n-1), \Omega^{\cdot}\right), \\
d(m, f)=(\partial m, d f+\alpha(m)) .
\end{gathered}
$$

Because of (4.1),

$$
d\left(C^{1}\right) \subset Z^{2}\left(\bigwedge^{\cdot} \mathscr{M}_{\text {top }}(n-2), \Omega^{\cdot}\right) \subset Z^{2}\left(\bigwedge^{\cdot} \mathscr{M}_{\text {top }}(n-1), \Omega^{\cdot}\right) .
$$

We have

$$
0 \rightarrow d\left(C^{1}\right) \rightarrow Z^{2}\left(\bigwedge^{\cdot} \mathscr{M}_{\text {top }}(n-1), \Omega^{\cdot}\right) \stackrel{t}{\rightarrow} \mathscr{W}_{n} \rightarrow 0
$$

exact, and

$$
X(n-2)=Z^{2}\left(\bigwedge^{\cdot} \mathscr{M}_{\text {top }}(n-2), \Omega^{\cdot}\right) \cap X(n-1)
$$

from which we deduce

$$
\operatorname{Ker}(t \mid X(n-1))=d\left(C^{1}\right) \cap X(n-1) \subset X(n-2) .
$$

With reference to $(6.13)$ we can now define

$$
\beta_{n}^{(1)}=t \circ \vartheta_{n}^{(1)}: \mathscr{P}_{n} \rightarrow \mathscr{X}_{n-1}
$$

We have $\partial \circ\left(\beta_{n}^{(0)}+\beta_{n}^{(1)}\right)=\partial \beta_{n}^{(0)}+\vartheta_{n}^{(1)}+\left(\partial \beta_{n}^{(1)}-\vartheta_{n}^{(1)}\right)=\left(\bigwedge^{2} \beta\right) \circ \partial+(p \circ s \circ t-1) \vartheta_{n}^{(1)}$. 
Define

$$
\vartheta_{n}^{(2)}=-(p \circ s \circ t-1) \circ \vartheta_{n}^{(1)}
$$

Arguing as above,

$$
\begin{aligned}
\vartheta_{n}^{(2)} & : \mathscr{P}_{n} \rightarrow X(n-3) ; \\
\beta_{n}^{(2)}=t & \circ \vartheta_{n}^{(2)}: \mathscr{P}_{n} \rightarrow \mathscr{X}_{n-2} ; \\
\partial \circ\left(\beta_{n}^{(0)}+\beta_{n}^{(1)}+\beta_{n}^{(2)}\right) & =\left(\bigwedge^{2} \beta\right) \circ \partial+\left(\partial \beta_{n}^{(2)}-\vartheta_{n}^{(2)}\right) \\
& =\left(\bigwedge^{2} \beta\right) \circ \partial+(p \circ s \circ t-1) \vartheta_{n}^{(2)} .
\end{aligned}
$$

This construction can be repeated until finally

$$
\partial \circ\left(\beta_{n}^{(0)}+\cdots+\beta_{n}^{(n-2)}\right)=\left(\bigwedge^{2} \beta\right) \circ \partial-\vartheta_{n}^{(n-1)} .
$$

But $\vartheta_{n}^{(n-1)}: \mathscr{P}_{n} \rightarrow X(0)=(0)$. (Note $\vartheta_{n}^{(n-2)}: \mathscr{P}_{n} \rightarrow X(1)$ and $\vartheta_{n}^{(n-1)}=$ $(p \circ s \circ t-1) \vartheta_{n}^{(n-2)}=0$, since $t \mid X(1)$ is injective.) Writing

$$
\beta_{n}=\beta_{n}^{(0)}+\cdots+\beta_{n}^{(n-2)}: \mathscr{P}_{n} \rightarrow \mathscr{X}_{2} \oplus \cdots \oplus \mathscr{X}_{n} \subset \mathscr{M}_{\text {top }}(n),
$$

we get $\partial \circ \beta_{n}=\left(\bigwedge^{2} \beta\right) \circ \partial$ completing the inductive step.

Remark (6.26). Note Image $(\beta) \subset \mathscr{M}_{\text {top }}$ is a $\mathbb{Q}$-vector space, and it seems natural to relate it to the $\mathbb{Q}$-minimal model of $V$. One has, of course,

$$
(2 \pi i)^{-1} \mathscr{P}(1) \subset \underset{U \subset V}{\lim } H^{1}(U, \mathbb{Q}) \text {. }
$$

Details remain to be worked out.

\section{BIBLIOGRAPHY}

1. A. A. Beilinson, Polylogarithm and cyclotomic elements, preprint.

2. A. A. Beilinson, A. B. Goncharov, V. V. Schechtman, and A. N. Varchenko, Aomoto dilogarithms, mixed Hodge structures and motivic cohomology of pairs of triangle on the plane, The Grothendieck Festschrift, Vol. I, Progr. in Math., vol. 86, Birkhäuser, Boston, 1990.

3. P. Deligne, Interprétation motivique de la conjecture de Zagier reliant polylogarithmes et régulateurs, preprint.

4. __ Le groupe fondamental de la droite projective moins trois points, preprint.

5. A. B. Goncharov, The classical trilogarithm, algebraic $K$-theory of fields, and Dedekind zeta-functions, Bull. Amer. Math. Soc. (N.S.) 24 (1991), 155-162.

6. J. Morgan, The algebraic topology of smooth algebraic varieties, Inst. Hautes Études. Sci. Publ. Math. 48 (1978), 137-204.

7. D. Sullivan, Infintiesimal calculations in topology, Inst. Hautes Études Sci. Publ. Math. 47 (1977), 269-331.

8. S. Bloch, Algebraic cycles and higher K-theory, Adv. in Math. 61 (1986), 267-304. 
9. __ Algebraic K-theory, motives, and algebraic cycles, Proc. Internat. Congr. Math. (Kyoto, 1990) (to appear).

10. P. Deligne and J. S. Milne, Tannakian categories, Hodge cycles, Motives, and Shimura Varieties, Lecture Notes in Math., vol. 900, Springer-Verlag, Berlin, 1982.

11. B. Totaro, Thesis, Univ. of Calif. at Berkeley, 1990.

Department of Mathematics, University of Chicago, Chicago, Illinois 60636 